\title{
Advantages of GNSS in Monitoring Crustal Deformation for Detection of Precursors to Strong Earthquakes
}

\author{
Guohua Gu, Wuxing Wang \\ Institute of Earthquake Science, China Earthquake Administration, Beijing, China. \\ Email: ggh@seis.ac.cn
}

Received December $13^{\text {th }}, 2012$; revised January $15^{\text {th }}, 2013$; accepted January $23^{\text {rd }}, 2013$

\begin{abstract}
Earthquake predictions in China have had rare successes but suffered more tragic setbacks since the Xintai earthquake in 1966. They have developed with twists and turns under the influence of the viewpoint that earthquakes are unpredictable etc. Though the Wenchuan earthquake of M8.0 in 2008 in China and the 2011 Tohoku earthquake of M9.0 in Japan were failed to predict, the GPS observations before and after these 2 events have shown that there were precursors to these events and large earthquakes were predictable. Features of different observation techniques, data processing methods are compared and some recent studies on precursory crustal deformations are summarized, so various advantages of GPS technique in monitoring crustal deformation are emphasized. The facts show that anomalies or precursors detected from GPS observations before the great Wenchuan earthquake have been the most remarkable results of explorations on crustal movements and earthquake precursors in China. GPS is in deed an excellent observation technique for earthquake prediction.
\end{abstract}

Keywords: Crustal Deformation; Earthquake Prediction; Precursor to Earthquake; Wenchuan Earthquake; Tohoku Earthquake in Japan; GPS; GNSS

\section{Introduction}

Explorations on earthquake prediction have been carried out for many decades in the world and for more than 40 years since the Xintai earthquake in 1966 in China. Though there have been quite rare successes, there have been more tragic setbacks in earthquake prediction in China. Are earthquakes predictable? Are crustal deformation observations, particularly GPS (Global Positioning System) observations, still useful and important monitoring techniques for earthquake prediction? These questions are not only concerned by the public and also they are fundamental problems that have puzzled the seismologists working on earthquake predictions.

The key to earthquake predictions is the detection of precursors to earthquakes. Based on the conception that earthquakes, particularly shallow earthquakes, are the result of tectonic crustal movements, so crustal deformation or movement monitoring has been the major technique for detection of precursors to earthquakes for quite a long time in the world. Therefore different techniques for crustal deformation observation have been tried to prove their usefulness in detecting precursors to earthquakes.

After the Xintai earthquake in 1966, some geodesists in China began researches on crustal deformation observations for earthquake prediction. Study on crustal deformation has been one of the main scientific disciplines for researches on earthquake prediction in China. During the 9th Five Year Plan about 150 million Yuan RMB was invested in the establishment of the Crustal Movement Observation Network of China (CMONOC) with GPS as the major observation technique and earthquake prediction as its main purpose. During the 11th Five Year Plan about 500 million Yuan RMB was invested in the establishment of the China Mainland Tectonic Environment Monitoring Network (CMTEMN) still with GPS as the major observation technique and earthquake prediction as its main purpose. CMTEMN is the continuation of CMONOC. At the time when the researches on earthquake prediction are in trough or depression, it is even more important to review the past explorations on crustal deformation observations and precursors to earthquakes, in order to learn experience and lessons from the practices and once again reiterate the important roles of crustal deformation observations and their scientific significance and promote more confidently new developments of earthquake predictions based on recent new discoveries from the observations in China. 


\section{Techniques of Crustal Deformation Observations}

In China the crustal deformation observations have been carried out through 2 major kinds of techniques based on their different features, that is, the techniques of continuous crustal deformation observations at permanent stations and the geodetic deformation observation techniques. The geodetic deformation observation techniques include various geodetic observation techniques that can be used in geodetic observations or even continuous observations on the earth surface to obtain the deformation results of a whole network in a large area from observation data with mathematical relations between them. The techniques of continuous crustal deformation observations at permanent stations include some geodetic observation techniques and techniques different from geodetic observation techniques, such as tiltmeters and strainmeters, which can be used to monitor repeatedly or continuously the crustal deformation in a very small area on the earth surface or under the ground or under the ocean, with deformation results mathematically independent among the stations.

\subsection{Techniques of Geodetic Crustal Deformation Observations}

During the period from the Xintai earthquake in 1966 to 1998, conventional geodetic observation techniques, such as leveling, EDM (Electronic Distance Measurement) and even triangulations were used for geodetic crustal deformation observations. Though the most advanced technology has been used in EDM and its precision has improved significantly, the relative precision of the baseline measurement has been lower than $10^{-6}$ at distances less than tens of kilometers and shorter. Precise leveling can be easily carried out and was the major technique used at that time and is still used now. The observation data of conventional geodetic survey, particularly the leveling data played important role in studies on the crustal deformations. The main disadvantages of conventional geodetic survey are labor intensive, low efficiency, low precision (except leveling), long observation duration, so it can not meet the urgent demand for short term or imminent earthquake predictions because it is impossible to detect anomalies or precursors in time. Therefore the conventional geodetic survey can only serve as an observation means for long and mid term earthquake predictions.

GPS has revolutionized the observation technique in modern Geodesy and become a powerful technique for crustal deformation observations. There are following advantages. The observation precision is high, with precision of $1 \mathrm{~mm}$ in horizontal component and $3 \mathrm{~mm}$ in vertical component as shown in statistics of the daily or weekly solutions in China or abroad [1], the relative precision can be $10^{-7}-10^{-8}$ for baselines of tens to hundreds of $\mathrm{km}$ and $10^{-9}$ for the baselines of thousands of $\mathrm{km}$; the results of GPS observations show long term stability; except on the ocean, the coverage of observation stations are global with different densities which are limited only by investment; rapid displacements and horizontal strain components of large amounts can be detected; both 1D vertical and 2D horizontal displacements and also all components of horizontal strains in areas of different scales can be obtained simultaneously; with dense stations it is easy to get pictures of the spatial developments of anomalous crustal deformations or deformation contaminations; observations of high sampling rate can be obtained for detection of short and imminent precursors to earthquakes; results of rapid and even real time data processing can be obtained for short and imminent earthquake predictions; in addition to the long and mid term precursory anomalous deformations, GPS can also detect imminent precursory variations in TEC (Total Electron Content) in the ionosphere; the costs for the establishment of the observation stations and observation operations are low. GPS is the technique for observations of the most comprehensive deformation components and is the best technique for crustal deformation observations at present that can meet different requirements for detecting precursory deformations in space, time and magnitude. Here the magnitude refers to the magnitudes of displacement and strain components, etc.

GPS has shown more and more clearly its advantages over other observation techniques. Because of these advantages, it was widely acknowledged that GPS can be used in monitoring crustal deformations and precursors to earthquakes with great expectations. Perhaps it can be used in earthquake early warning system. The establishment of CMONOC in 1998 marked the beginning of the new phase of exploration on crustal deformation observations and precursors to earthquakes in China.

The main disadvantages of leveling are the long observation duration and inability to get seasonal variations at the benchmarks. Leveling shows advantages of high precision and high density of the benchmarks in small areas. Studies show that for detection of vertical deformations in large areas, such as over Chinese mainland, GPS observations should replace leveling. The reason is obvious. Corrections for troposphere delays and earth tide displacements can be performed for every observation in GPS data processing but can not be made in leveling data processing. The leveling is carried out through a series of setups of the level instrument in the lowest part of the troposphere with most serious refraction and the leveling at each setup of the level only takes a few minutes. The accumulation of these errors in leveling can be at the $\mathrm{dm}$ level along the leveling routes from the east 
to the west on Chinese mainland. In contrast, the vertical positions at GPS stations are determined independently with precision of $3 \mathrm{~mm}$ by observations for a few days [1].

GLONASS of Russia, Beidou of China, Galileo of Europe and GPS of the United States have jointly formed GNSS (Global Navigation Satellite System). The multiple GNSS will significantly improve the precision of positioning, particularly, because of the doubled number of GNSS satellites, the precision of single epoch positioning will improve significantly, so it will benefit the acquisition of the short term or imminent crustal deformations precursory to large earthquakes.

Though great progress has been achieved in satellite gravity measurements, their precision and spatial resolution still can not meet the demand expected for earthquake predictions and they can not replace leveling at present. Coseismic changes of huge earthquakes in the gravity field have been observed by satellite gravity measurements, even with their global coverage over the oceans.

InSAR (Interferometric Synthetic Aperture Radar) has been an important achievement in space technology for the earth observations after GPS. The main advantage of InSAR is that the images of the crustal deformations are spatially continuous in a certain area on the land and they have played significant roles in the studies of the coseismic vertical deformations of great earthquakes that occurred on the land. But the most obvious disadvantages of InSAR are its low precision and low frequency of image acquisition, so it has not been used in monitoring deformations precursory to earthquakes.

\subsection{Techniques of Continuous Crustal Deformation Observations at Permanent Stations}

Continuous observations and high precision of crustal deformation observations are necessary for short term and imminent earthquake predictions. In order to explore earthquake predictions, especially the short term and imminent earthquake predictions, techniques of continuous crustal deformation observations at permanent stations, such as tiltmeters and strainmeters etc., have been used in Russia, Japan and US, much earlier than the use of GPS, in order to overcome the disadvantages of low precision, long sampling intervals and observations that can only be carried out on the earth surface in conventional geodetic surveys. Since the Xintai earthquake in 1966 these techniques also have been used in China. The instruments of these techniques have developed from analog to digital recording and the data transfer has been greatly improved with the development of technology in telecommunications. The instruments of high precision can record solid earth tides, for example the precision of tiltmeters and strainmeters can be better than $10^{-8}$. The theoretical data of the solid earth tides can be used to identify the anomalous crustal deformations from solid earth tide observations. Though the technology used in the development of the instruments for continuous crustal deformation observations has been quite advanced and the data quality has been greatly improved, the instrument precision or sensitivity always contradicts to the stability of the observation results because of the existence of environmental contaminations, such as the contaminations from the atmospheric pressure, ground water level, temperature and pressure at the depths. Environmental contaminations appear in almost all observations, the higher the observation precision, the more venerable to contaminations, the less the stability of observation results or the shorter the stability periods. In order to avoid or mitigate contaminations at stations of continuous crustal deformation observations, durable observation devices are installed in tunnels or boreholes at some depths at high construction costs. Therefore the number of these stations is still quite small. In addition, the measurement ranges of the instruments of high precision and sensitivity are limited. For example, the higher the precision or the sensibility of the tiltmeters, the smaller the tilt measurement range, so it is impossible for the tiltmers to detect large tilts precursory to large earthquakes or large coseismic tilts.

Though there have been reports about distortions of the solid earth tides from their theoretical values imminently before some great earthquakes, the observed phenomena have to be confirmed with more data. Leveling and baseline measurements of conventional geodetic techniques at short distances detected anomalous deformations of long and short terms before several great earthquakes in China, though solid earth tides were not recorded. Therefore observations of solid earth tides can only be one aspect for studies on crustal deformations for earthquake predictions and should not be considered as the standard requirement for the precision of crustal deformation observations for earthquake predictions.

Because of low density of permanent stations of continuous crustal deformation observations and complicated contaminations, it is difficult or even impossible to detect the spatial influence and development of the anomalous deformations or deformation contaminations through analysis on the changes at stations of distances of hundreds of kilometers apart.

Continuous crustal deformation observations at permanent stations and GPS observations are technically quite different, but there is one common feature that both observations can be carried out continuously and the single epoch GPS solutions, like some continuous crustal deformation observations, can also record seismic waves 
near the epicenters of large earthquakes.

Continuous crustal deformation observations at permanent stations started much earlier than GPS, but there have been only some scattered results of precursory or anomalous crustal deformations because of above mentioned limitations. Though studies on continuous crustal deformation observations for earthquake prediction have advanced slowly, they should be carried out with more efforts.

In contrast, studies on GPS observation results in more than 10 years in China show that the advantages of GPS observations and the inseparable relationship between the tectonic crustal deformation and the occurrence of earthquakes have led to remarkable results of deformation anomalies or precursors to earthquakes.

\subsection{Networking of Crustal Deformation Observations}

Besides observation instruments, crustal deformation observations should be realized in a large area through careful selection of the observation sites with adequate density and suitable site configurations and locations of tectonic significances, the durable and stable monuments, continuous or repeated observations of reasonable cycles for a long period, sampling rate of observation data, auxiliary observations, data transfer and processing etc. These are problems of the networking of crustal deformation observations. For the matured techniques for crustal deformation observations, the main concern is the density of the observation sites, the denser the better. Obviously, it is ideal to obtain deformation pictures continuously both in time and space.

Rather favorable observation networks can be established on Chinese mainland for monitoring crustal deformations and detecting and identifying precursors to earthquakes because of the unique tectonic environment of intensive crustal movements and the inland strong earthquakes that occurs with relatively low frequency in time and relatively large separation in space. The establishment of the GPS observation networks in China has fundamentally changed the crustal deformation observations and a large amount of valuable observation data has been accumulated timely for the studies.

\section{Studies on Data Processing of Crustal Deformation Observations}

The timely data processing and analysis are one of the key links in earthquake predictions. Crustal deformations and the precursors can only be obtained through studies on the spatial and temporal features or characteristics of the crustal deformations and the contaminations from observations for a relatively long period and in a quite large area. For many years, Chinese geodesists have fur- ther developed the data processing and analysis of crustal deformation observations by applying data processing techniques and models used in conventional geodesy and GPS space geodesy.

\subsection{Data Processing for Acquisition of the Crustal Deformations}

There are mainly 2 parts in the data processing of crustal deformation observations: the adjustments of the observation data and analyses of crustal deformations from the results of the previous adjustments. In comparison to the most complicated data processing for GNSS and other space geodetic techniques, the data processing of the continuous crustal deformation observations and the conventional geodetic observations is quite simple. Software packages from the United States and Europe have been used in automatic GPS data processing to get rapid results of high precision. GNSS data processing software packages, such as PANDA and other software packages have been developed in China and will be used for monitoring crustal deformations. Though different software packages share similar functions, each of them has its own advantages and can meet different purposes in different applications. GNSS data processing has become the basic skill that is not only necessary for processing the observation data but also considerably benefits the researchers in understanding and proper use of GNSS data processing results.

In studies on earthquake predictions, time series and spatial patterns of crustal deformations should be obtained to identify precursors and for further analysis. There are various data processing methods and deformation models for explanation. Here the problem is the selection of useful methods or models. In the studies, solid basic theories or methods must be used to get the most reliable and valuable results. For example, the most reliable precursory crustal deformations can be identified from the time series of displacements at individual sites and strain components in Delaunay triangles formed by GPS sites. Based on the original and discrete numerical results of individual stations or Delaunay triangles, analyses on the time series or spatial patterns of deformation components through harmonic approximation, statistics or modeling can show even more strikingly the anomalies in area, time interval, frequency domain and spatial pattern. Among various deformation components, the displacement fields and the horizontal strain fields are two of the most important aspects in the analysis on the crustal deformations.

\subsection{Analysis of Displacements and Strain Components}

Though GNSS can get 3 dimensional positions on the 
earth surface, the 3D deformations on the earth surface are usually decomposed into $1 \mathrm{D}$ vertical deformations and 2D horizontal deformations on the ellipsoid because of their different characteristics in temporal variations [2-4]. Except 3D crustal deformation observations carried out at the depths under the earth surface, any 3D geodetic deformation surveys carried out on the earth surface can only be used to get 2D strains on certain reference surfaces, usually on the ellipsoid, the sphere or the plane. Repeated GNSS observations at 3 stations of favorable geometric configurations can perform as a strainmeter and all components of the horizontal strains can be obtained along with horizontal displacements at the same time.

In the analysis of the results of the crustal deformations obtained from geodetic techniques, such as GNSS, the basic theory on the analysis of the displacement fields is the theory of free network adjustments usually performed through similarity transformations and the most important results from the adjustments are the displacements in regional reference frames [5-7]. The horizontal displacement fields in the regional reference frame obtained by subtracting the regional Euler rotation vector are also realized through similarity transformation on the sphere. Mathematically there can be multiple solutions from similarity transformations with respective reference frames and some of the solutions, particularly the solutions of horizontal displacements, are quite different from each other and therefore their explanations can be quite different. It is well known that there are also multiple mathematical solutions for the motion of celestial bodies and only some of them with proper stable reference frames are physically meaningful for the explanations on the satellite motions in the earth system, the planet motions in the solar system and the star motions in the galaxy. The global reference frames [8] are always needed in GNSS data processing and are reference frames used in studies on the global plate tectonic motions. The model of global plate tectonic motions is an important element of the global reference frames. The regional reference frames are more meaningful both in Physics and Tectonics and appropriate in explaining regional relative crustal movements [5-7] and have been used in different countries or regions. The differences between 2 displacement solutions, particularly between 2 horizontal displacement solutions in the global and regional reference frames are quite significant in some regions. The horizontal displacement fields can give a clear picture of the mutual interactions of different plates or blocks and the origins of the strong earthquakes. The displacement time series at GNSS stations far from the epicenters of some huge earthquakes, obtained in the regional reference frames, have shown clearly that the coseismic horizontal displacements are the elastic re- bound of the preseismic displacements and they are strong evidences of the existence of precursory crustal deformations $[9,10]$.

The strain components obtained from horizontal displacements can show clearly differences in crustal deformations or stresses in different parts of the crust. Analyses on strain components, particularly the components of shear strains, are of important significance in earthquake predictions because shear stresses are directly responsible for the earthquake occurrences. The equations of derivatives used in strain calculations can be deduced on the plane, the sphere and the ellipsoid. All their mathematical expressions are formally identical. In the numerical calculations, the approximate values of the derivatives on the plane, the sphere and the ellipsoid are in general in good agreement; the smaller the areas with stations used in strain calculations, the better the agreements of different calculations. The advantage of strain calculations in different ways is mainly exhibited in the convenience of calculations in different extents. Strain calculation on the ellipsoid perhaps is the most convenient because data conversions to the plane or the sphere can be omitted.

Analysis on the time series of GNSS results shows that there are periodic annual variations in vertical displacements at a number of GPS stations [2-4], which obviously complicate the data analysis. The reason for the annual variations remains unknown or only known in some degree. Because of different features of the vertical and horizontal displacements, it is reasonable to analyze them separately, even with different regional reference frames.

\subsection{Contaminations and Deformation Models}

Contaminations in crustal deformations are quite common and are one of the major problems in data analysis. One of the most serious contaminations is the ground subsidence caused by ground water withdraw of large volumes, particularly in the developed regions in east China with increasing subsidence areas. Such subsidence of large amount can be easily identified but it can not be analyzed quantitatively because the changes in subsidence are governed by complicated factors. The regions of subsidence can only be excluded in the data analysis at present.

Results of GPS observations have shown that in comparison to the vertical crustal movements the horizontal crustal movements are dominant and the plate tectonic motion model is a useful macroscopic model in studies on the relationship between the crustal movements and the occurrences of strong earthquakes.

Theoretical models are mainly the approximations for explanation of the observed complicated facts in different 
degrees. Based on the dislocation models, the general feature and parameters of an earthquake fault can be estimated from coseismic deformations and the post seismic deformations near the epicenter can be simulated. Though dislocation models etc. have been used in studies, it is difficult to explain some special or exceptional deformations, such as coseismic displacements at individual stations. These exceptional deformations could be clues for improving the models or creation of new models. Till now no numerical models can be used to explain the preseismic crustal deformations or movements. It is the highest expectation for the seismologists to get the mathematical and physical models for the precursory crustal deformations. It is believed that only when these models are available, the earthquake prediction will be based on solid scientific foundations.

Because of above mentioned advantages, crustal deformation results from GPS observations, particularly continuous GPS observations, are reliable for studies on earthquake predictions.

\section{Studies on the Crustal Deformation Precursors to Earthquakes}

Though great progress has been achieved in crustal deformation observations, obviously the most difficult problem in studies on precursors to earthquakes is the rather rare opportunities for their detection. Precursory crustal deformations or anomalies can only be detected and identified in suitable observation networks with suitable observation techniques for long term observations carried out in a much large area around the epiceneters during proper time periods before earthquakes.

\subsection{From First Few Successes to Tragic Setbacks and "Earthquakes Are Unpredictable"}

From the Xintai earthquake in China in 1966 to 1998, the Haicheng earthquake in 1975, the Tangshan earthquake in 1976 and several other earthquakes occurred in China. The result of vertical deformation obtained from leveling contributed in certain degree to the successful prediction of the Haicheng earthquake. Though the failure in predicting the Tangshan earthquake was a hard blow to the researches on earthquake prediction in China, the crustal deformation observations accumulated some precursory deformation data, including repeated leveling data and data of continuous crustal deformations at some observation stations. The crustal deformation observations during this period were the earliest and meaningful trials in explorations on earthquake predictions in China.

During the period when Japan, United States and China started to use GPS in explorations on earthquake predictions with great expectations, GPS observations at rather dense stations in US and Japan did not detect crustal deformations precursory to several earthquakes of magnitudes above 7.0 occurred in GPS networks. So there has been a prevailing viewpoint that earthquakes are not predictable. GPS has not played its role in earthquake prediction in the developed countries and the reasons should be studied; therefore it is hard for scientists to imagine that rather limited GPS observations in China could play any role in earthquake predictions. There were doubts on the actual precision in crustal deformation observations achieved by GPS and even doubts on the existence of the crustal deformations or their magnitudes that can be detected during the preparation of a strong earthquake. The predictions of both the huge Wenchuan earthquake of M8 on May 12, 2008 and the giant Tohoku earthquake of M9.0 in Japan on March 11, 2011, failed, though large amount of fund for earthquake predictions was invested on GPS observations both in China and Japan. The failures seem to confirm that GPS is invalid in monitoring crustal deformations for earthquake predictions and they seem to announce the dissolution of the hope in earthquake predictions.

\subsection{The First and Unique Results in Exploration on Precursors to Earthquakes Obtained from GPS Observations}

However, studies [9-15] on the results of GPS observations were carried out before and after Wenchuan earthquake of M8 and the Tohoku earthquake of M9.0 in Japan. The results have shown that there were crustal deformations precursory to the earthquakes and earthquakes are predictable at least for earthquakes of comparable magnitudes.

There were 1000 regional GPS stations in CMONOC (Crustal Movement Observation Network of China) with dense GPS stations in North China and the North-south seismic belt, where the epicenter of the Wenchuan earthquake was located. 4 GPS observation campaigns at regional stations were carried out in 1999, 2001 (finished before the Kunlun mountain earthquake of M8.1 in 2001), 2004 and 2007. Each campaign lasted about 6 months and each station was occupied at least for 4 days. 25 fiducial stations on Chinese mainland and several IGS (International GPS Service) stations in the neighborhood of China have accumulated data of continuous GPS observations for more than 10 years. There are also stations of continuous GPS observations near the epicenter of the Wenchuan earthquake, established by the Sichuan Seismological Bureau, and observations at these stations started several months before the event. All these GPS observations provide abundant data for studying the crustal movements before the Wenchuan earthquake.

Time series of horizontal displacements at the fiducial stations of continuous GPS observations showed obvious 
anomalies in early 2008 in a quite large area before the great Wenchuan earthquake [9].

The GPS observations at the regional stations in 2007 were processed before the Wenchuan earthquake. The data processing result was available to the author 10 days after the Wenchuan earthquake. In 3 days after the result was available, the preseismic anomalies in the first shear strain near the epicenter of the Wenchuan earthquake were obtained and analyzed based on the trend surface mapping developed in GMT [16] and used in our previous studies. The first shear strain represents left lateral shear in $\mathrm{N} 45^{\circ} \mathrm{E}$ or right lateral shear in $\mathrm{N} 45^{\circ} \mathrm{W}$ under north-south compression and east-west extension. The area with the anomalies was unique on Chinese mainland before the event. Subsequent detailed studies have shown clearly the development of the anomalies of increasing magnitudes and in an area increasing in size $[11,12]$. The anomalies became significant in 2004. The area with anomalies shown in the result from 1999 to 2007 was about 1 million square kilometers with about $1 / 3$ of regional GPS stations.

In recent years progress has been made in GPS data processing for single epoch solutions and seismic waves from some great earthquakes have been obtained from GPS observations of high sampling rates, such as once per second. Based on this progress, in early 2009 single epoch solutions of 30 s sampling rate were obtained with BERNESE software from continuous GPS observations at 4 stations near the epicenter of the Wenchuan earthquake. Single epoch solutions show that within one hour before the great Wenchuan earthquake 3 stations subsided quickly with large amount at the same time and at the PIXI station, $36 \mathrm{~km}$ from the epicenter, the largest subsidence was about $300 \mathrm{~mm}[13,14]$. The precision of the single epoch solutions is 10 times lower than the precision of the daily or weekly solutions, but GPS can detect such rapid and large (vertical) displacements.

At the end of 2010 the vertical displacements at regional stations were reanalyzed with trend surface mapping. The mapping results show that there were also anomalies in vertical displacements near the epicenter [15] and they also became significant in 2004.

The far field coseismic displacements, particularly the coseismic horizontal displacements are recognized as the most obvious and reliable crustal movements associated with the occurrence of the earthquakes. The time series of horizontal displacements in the regional reference frame on the Chinese mainland show that the coseismic horizontal displacements of the Wenchuan earthquake are the elastic rebound of the preseismic displacements and are the evidences that the preseismic anomalies in horizontal displacements were precursors to the event [9]. In comparison to the previous studies on coseismic horizontal displacements, the key to this understanding relies on the time series obtained in the regional reference frame.

In addition, imminent anomalies in TEC of the ionosphere were also detected by GPS observations before the earthquake [17].

GPS observations before the Wenchuan earthquake created several records that have been the first in the history of monitoring precursors to the earthquakes. The great Wenchuan earthquake was the first earthquake of M8.0 with long term, mid term, short and imminent preseismic anomalies detected by GPS. Among the observation techniques for earthquake prediction, GPS is the unique observation technique that detected long term, mid term, short term and imminent anomalies before the great Wenchuan earthquake. The anomalies could be used in predicting the location, magnitude and occurrence time of the earthquake. The facts show that GPS observation technique is not only useful in long term and mid term predictions but also in short term and imminent predictions because of its various advantages in detecting crustal deformations.

\subsection{One More Rare Opportunity for the Exploration of Precursors to Earthquakes}

The Tohoku earthquake in Japan did not occur inside the network of continuous GPS observations in Japan but the GPS station nearest to the epicenter is only about $100 \mathrm{~km}$ away. Even though no preseismic results from this network are available, this event has been a rare opportunity to verify the studies on the horizontal displacements before and after the great Wenchuan earthquake. In addition to the stations in Japan, far field coseismic horizontal displacements were also observed for the event on Chinese mainland, in south Korea and in central Asia. The coseismic horizontal displacements are the elastic rebound of the preseismic of this event and they show that there were crustal movement precursors to this event [10]. Anomalies on Chinese mainland and in central Asia were identified before the earthquake; however it was impossible to make prediction based only on these anomalies. In addition, imminent anomalies in TEC of the ionosphere were also detected by GPS observations before the earthquake.

Based on reliable results from observations it was concluded that the precursory crustal movements should have been observed in the GPS network in Japan before the earthquake [10]. Recent studies by the Japanese scientists show that "The preceding aseismic and coseismic slip decreased the coupling rate off the Tohoku coast, suggesting the possibility that the preceding slip represented a precursive stage of the Tohoku earthquake" [18].

Available observation results, including results of both continuous deformation observations at permanent sta- 
tions and GPS observations show that the displacement or deformation processes before and after the strong earthquakes are quite similar to deformation processes before and after the failure shown in the rock sample tests. They are laboratory evidences and clues for the studies on the precursory process during the preparation of an earthquake for earthquake prediction.

The significant horizontal displacements in a vast area before the Wenchuan and Tohoku earthquakes in Japan show that they were originated from the plate tectonic motions.

\section{Conclusions}

As a geodesist working on earthquake prediction researches for many years, I have participated in the development of crustal deformation observations and earthquake prediction researches in China and witnessed the its twists and turns. I worked on the data analysis and predictions before the Tangshan and Wenchuan earthquakes and carried out a lot of researches after these events. Though there were tragic setbacks in earthquake predictions, they mean neither the failure of observations for earthquake predictions nor the end of explorations on earthquake predictions. Experience and lessons in the past should be summed up and will be instrumental to the development of earthquake predictions in China in the future.

\subsection{GNSS Is Still One of the Main Pillars of Observation Techniques for Earthquake Prediction}

Results from crustal deformation observations, particularly from GPS observations, have indicated that there have been crustal deformations precursory to earthquakes. Various anomalies or precursors to the great Wenchuan earthquake detected by GPS observations have been the most remarkable and valuable results of crustal deformation observations and explorations on earthquake predictions in China. The existence of precursors to earthquakes is a fact that can not be denied and is the practical foundation for earthquake predictions. The crustal deformation observations still remain as a useful approach and GNSS is one of the main pillars of observation techniques for earthquake prediction. Various advantages and characteristics of GNSS observations, particularly, various anomalies or precursors to great earthquakes detected by GPS observations have manifested that GNSS observation should be further developed and related studies should be strengthened.

Systems of crustal deformation observations or other observations should be improved on the basis of previous explorations of earthquake prediction by applying more advanced techniques that can detect various precursors for earthquake predictions. Densification of continuous GNSS observation stations are expected for earthquake prediction in China.

\subsection{Earthquake Prediction Is a Task Based on Long Term and Patient Researches}

Disasters of great earthquakes are devastating; therefore earthquake prediction is an urgent demand of the society and predictions of great earthquakes are the responsible duty of the seismologists working on earthquake predictions. Above mentioned facts show that earthquake predictions are possible in different degrees even at present. Though the region where a great earthquake is preparing could be quite large in area, the probability of its occurrence is quite small and there are problems of the monitoring networks, so there are quite rare opportunities to detect and study crustal deformation precursors of small changes. It is still a dream of the seismologists to get complete temporal and spatial pictures of crustal deformations and other phenomenon during the process of preparations of great earthquakes of different magnitudes and different types. Researches on earthquake predictions remain the difficult tasks for a rather long time.

Researches on earthquake predictions have combined researches in different disciplines of seismology, geodesy, geomagnetism and other branches in geophysics that have developed for a rather long time and their basic theories and methods have been verified and perfected time and again. These theories and methods should be applied properly to solve new problems in earthquake predictions. The data processing and analysis in time are the key link in earthquake predictions. Comprehensive studies of observation results from the different disciplines of science are the natural trend and an extremely difficult task in the development of earthquake predictions.

International cooperation is necessary for predicting huge earthquakes. The unique tectonic environment in China is favorable for detecting precursors to earthquakes; it is quite possible that the breakthrough in earthquake prediction can be realized first in China.

\section{Acknowledgements}

This research is funded by Research Fund for Senior Scientists from China Earthquake Administration.

\section{REFERENCES}

[1] G. Blewitt, "GPS and Space-Based Geodetic Methods," Elsevier, Amsterdam, 2007, p. 352.

[2] D. Dong, P. Fang, Y. Bock, et al., "Anatomy of Apparent Seasonal Variations from GPS Derived Site Position Time Series," Journal of Geophysical Research, Vol. 107, 
No. B4, 2002. doi:10.1029/2001JB000573

[3] G. Blewitt and D. Lavalle, "Effect of Annual Signals on Geodetic Velocity," Journal of Geophysical Research, Vol. 107, No. B7, 2002. doi:10.1029/2001JB000573

[4] G. H. Gu, "Time Series of Vertical Displacements Obtained at Fiducial Stations of Continuous GPS Observations," Seismogeology, Vol. 27, No. 2, 2005, pp. 332-340.

[5] G. H. Gu, X.-H. Shen, M. Wang, et al., "General Characteristcs of the Recent Horizontal Crustal Movement in Chinese Mainland," Acta Seismologica Sinica, Vol. 14, No. 4, 2001, pp. 384-393. doi:10.1007/s11589-001-0116-1

[6] G. H. Gu, Y. Fuand and W. X. Wang, "Horizontal Crustal Movement in Chinese Mainland from 1999 to 2001," Acta Seismologica Sinica, Vol. 17, 2004, pp. 53-60.

[7] G. Blewitt, "Overview of the SNARF Working Group, Its Activities, and Accomplishments," 8th SNARF Workshop AGU Fall Meeting, San Francisco, 11 December 2007.

[8] J. Ray, D. Dong and Z. Altamimi, "IGS Reference Frames: Status and Future Improvements," Position Paper at IGS 2004 Workshop, Berne, 1-5 March 2004.

[9] G. H. Gu and W. X. Wang, "Coseismic Displacements of the Great Wenchuan Earthquakes in Sichuan Detected by GPS Observations," Earthquake, Vol. 29, No. 1, 2009, pp. 92-99.

[10] G. H. Gu and W. X. Wang, "Far-Field Crustal Movements before and after the 2011 Ms9.0 Japan Earthquake from GPS Observations," Geodesy and Geodynamics, Vol. 2, No. 3, 2011, pp. 1-7.

[11] G. H. Gu, W. X. Wang, G. J. Meng and Y. R. Xu, "Crustal Movements before and after the Great Wenchuan Earthquake Observed by GPS," Geomatics and Information Science of Wuhan University, Vol. 34, No. 11, 2009, pp.
1336-1339.

[12] G. H. Gu, W. X. Wang, Y. R. Xu and W. J. Li, "Horizontal Crustal Movement before the Great Wenchuan Earthquake Obtained from GPS Observations in the Regional Network," Earthquake Science, 2009, Vol. 22, No. 5, 2009, pp. 471-478.

[13] G. H. Gu, G. J. Meng and Y. Fang, "Crustal Movements in the Earthquake Area before and after the Great Wenchuan Earthquake as Detected by Precise Single Epoch Positioning of GPS Observations," Acta Seismologica Sinica, Vol. 33, No. 3, 2011, pp. 319-326.

[14] G. H. Gu, G. J. Meng and W. X. Wang, "Anomalous Crustal Movements before the Great Wenchuan Earthquake Obtained from GPS Observations," Geodesy and Geodynamics, Vol. 2, No. 2, 2011, pp. 13-22.

[15] G. H. Gu and W. X. Wang, "Vertical Crustal Movement before and after the Great Wenchuan Earthquake Obtained from GPS Observations in the Regional Network," Earthquake, Vol. 31, No. 3, 2011, pp. 1-8.

[16] P. Wessel and W. H. F. Smith, "New, Improved Version of Generic Mapping Tools Released," Eos, Transactions American Geophysical Union, Vol. 79, No. 47, 1998, p. 579. doi:10.1029/98EO00426

[17] J. Y. Li, G. J. Meng, M. Wang, H. Liao and X. H. Shen, "Investigation of Ionospheric TEC Changes Related to the 2008 Wenchuan Earthquake Based on Statistic Analysis and Signal Detection," Earthquake Science, Vol. 22, No. 5, 2009, pp. 545-553. doi:10.1007/s11589-009-0545-9

[18] S. Ozawa, T. Nishimura, H. Munekane, et al., "Preceding, Coseismic, and Postseismic Slips of the 2011 Tohoku Earthquake, Japan," Journal of Geophysical Research, Vol. 117, No. B17, 2012. doi:10.1029/2011JB009120 